\title{
Low virulent infectious salmon anaemia virus (ISAV-HPR0) is prevalent and geographically structured in Norwegian salmon farming
}

\author{
Trude M. Lyngstad ${ }^{1, *}$, Anja B. Kristoffersen ${ }^{1,2}$, Monika J. Hjortaas ${ }^{1}$, \\ Magnus Devold ${ }^{3}$, Vidar Aspehaug ${ }^{3}$, Rolf B. Larssen ${ }^{4}$, Peder A. Jansen ${ }^{1}$ \\ ${ }^{1}$ Norwegian Veterinary Institute, PO Box 750 Sentrum, 0106 Oslo, Norway \\ ${ }^{2}$ University of Oslo, Department of Informatics, PO Box 1080, Blindern, 0316 Oslo, Norway \\ ${ }^{3}$ PatoGen Analyse AS, PO Box 1527, 6025 Ålesund, Norway \\ ${ }^{4}$ Norwegian School of Veterinary Science, PO Box 8146 Dep, 0033 Oslo, Norway
}

\begin{abstract}
Infectious salmon anaemia (ISA) is a severe disease in farmed Atlantic salmon Salmo salar that has caused epidemic outbreaks in most salmon-producing countries worldwide. The disease is caused by virulent ISA virus (ISAV). Low virulent variants of the virus, characterised by a full-length sequence in the highly polymorphic region of segment 6 in the virus genome, have been reported with increasing frequencies. These variants of the virus, termed HPR0, have been proposed to be ancestors of virulent ISAV. We examined this idea through studies of the phylogeographic and environmental distribution of ISAV-HPR0, as well as phylogeographic associations between virulent ISAV and ISAV-HPR0. Samples from 232 fish groups were screened for ISAV. Real-time RT-PCR was used for detection of ISAV, and the ISAV haemagglutinin esterase (HE) gene was characterised for positive samples. A Mantel test was used to test phylogeographic associations between pairs of ISAV-HPR0 HE gene sequences. A rank test was used to test associations between HE gene sequences from virulent ISAV and ISAV-HPR0. ISAV-HPR0 was detected in fish groups both in freshwater and marine environments, and in juveniles, on-grown marine salmon and broodstock salmon. Genetic and geographic distances between pairs of ISAV-HPR0 HE gene sequences were positively correlated, suggesting that the population of ISAV-HPR0 is geographically structured. Finally, we found a spatial association between fish groups with virulent ISAV $(\mathrm{n}=21)$ and fish groups with ISAV-HPR0 $(\mathrm{n}=27)$, supporting the hypothesis that ISAV-HPR0 may undergo a transition to virulent ISAV.
\end{abstract}

KEY WORDS: Infectious salmon anaemia virus $\cdot$ ISAV $\cdot$ HPR0 $\cdot$ Atlantic salmon $\cdot$ Norway

\section{INTRODUCTION}

Infectious salmon anaemia (ISA) is a severe disease in farmed Atlantic salmon Salmo salar that has caused epidemic outbreaks in most salmon-producing countries worldwide (Office International des Epizooties 2009). The disease is caused by the ISA virus (ISAV) within the family Orthomyxoviridae (Kawaoka et al. 2005).
ISAV has a genome consisting of 8 segments (Mjaaland et al. 1997), of which segment 6 encodes the haemagglutinin esterase (HE) gene (Krossøy et al. 2001, Rimstad et al. 2001, Falk et al. 2004). The HE gene consists of an N-terminal part, a transmembrane domain and a C-terminal part. A small highly polymorphic region (HPR) is located upstream of the transmembrane region (Devold et al. 2001, Mjaaland et al. 2002). It has been proposed that the polymor- 
phism in the HPR of virulent ISAV HE genes could be explained by a differential deletion model (Mjaaland et al. 2002). These authors suggested that a longer ancestral sequence, stretching over a 130 nucleotide long region (HPR0), represents a variant from which virulent ISAV with deletions in the HPR may arise. Shortly after, a variant with a corresponding long gene sequence was reported from an apparently healthy specimen of wild Atlantic salmon in Scotland (Cunningham et al. 2002). Detection of ISAV by RTPCR from wild and farmed salmon without clinical signs of ISA (Raynard et al. 2001, Cook-Versloot et al. 2004, Plarre et al. 2005) further supported the notion that some ISAV strains may be of low virulence. The first reports on the putative ancestral low virulent ISAV (ISAV-HPR0) sequences were followed by numerous findings of ISAV-HPR0 variants from the Faroe Islands, Norway and Scotland, all from apparently healthy fish, supporting low virulence of ISAVHPR0 (Nylund et al. 2007, McBeath et al. 2009, Christiansen et al. 2011, Lyngstad et al. 2011).

The driving forces behind transitions between virulent and low virulent variants of ISAV are not known. ISAV-HPR0 has not been isolated in cell culture, and a direct link between ISAV-HPR0 and virulent ISAV remains to be demonstrated. Nevertheless, deletion events are more likely to occur than inserts. The presence of different HPR deletion patterns in otherwise highly similar ISAV sequences strongly supports a model of several independent deletions, rather than insertion events (Mjaaland et al. 2002, Nylund et al. 2003).

The occurrence of ISA in Norwegian salmon farming in recent years has been described as partly spatio-temporal clusters of farms with disease outbreaks and partly outbreaks in farms isolated from apparent sources of infection. The local clusters have been explained by horizontal spread of infection between proximate farms, whereas the isolated outbreaks have had an unknown source of infection (Aldrin et al. 2011). It has been suggested that ISA may arise from spontaneous transitions of ISAV-HPR0 to virulent ISAV (Cunningham et al. 2002, Mjaaland et al. 2002, Nylund et al. 2003) and that this may cause either solitary outbreaks or local epidemics through local transmission (Lyngstad et al. 2011). Here we examined this idea through studies of the phylogeographic and environmental distribution of ISAVHPR0, as well as phylogeographic associations between virulent ISAV and ISAV-HPR0. Since we provide data supporting the scenario of spontaneous transitions of ISAV-HPR0 to virulent ISAV causing disease outbreaks, we also suggest estimates of the annual risk of isolated ISA outbreaks arising from transitions of ISAV-HPR0.

\section{MATERIALS AND METHODS}

\section{Study material}

The study material consisted of 2 separate datasets: (1) data on ISAV screening of fish groups of farmed Atlantic salmon (screening data), and (2) data on genetic characterisation of the ISAV HE gene from fish groups of farmed salmon with confirmed outbreaks of ISA (outbreak data). The fish groups included in the screening data were not under suspicion of being ISAV infected.

A fish group was defined as the fish stock at a site at the time of sampling. Fish groups at a given site separated by a fallowing period were counted as different fish groups. The fish groups consisted of either juvenile salmon sampled in freshwater, on-grown marine salmon or broodstock salmon sampled either in marine environments or shortly after relocation back to freshwater pre-reproduction. Sampling and data collection were performed in the period from 2006 to 2010.

The case definition of an ISA outbreak in a fish group was provided in the contingency plan for ISA in Norway (Norwegian Food Safety Authority 2007) and was based on clinical signs and post mortem findings consistent with ISA, as described in the OIE Manual of diagnostic tests for aquatic animal diseases (Office International des Epizooties 2009). A fish group (and thus also the site) with ISA retained the status of infected until all fish were removed from the site.

In order to investigate whether there was any geographic structure in the distribution of ISAV-HPR0 genotypes in Norway, we made use of existing organ samples that were collected through projects with a broad geographical spread of the samples (Fig. 1). The screening data comprised samples from 5 collaborating projects with varying sampling protocols: a cohort study of pancreas disease (Jansen et al. 2010), a case-control study of cardiomyopathy syndrome (B. Fineid pers. comm.), the 'Stans spredning av PD til Midt-Norge (Stop the spread of PD to Mid-Norway)' project (A. Østvik pers. comm.), the 'Tracing ISA' project (Lyngstad et al. 2011) and a pilot study on broodstock fish groups performed in 2008 by the Norwegian Food Safety Authority. All together, the screening data included results from real-time RT-PCR screening for ISAV from 232 fish groups covering 210 farm 


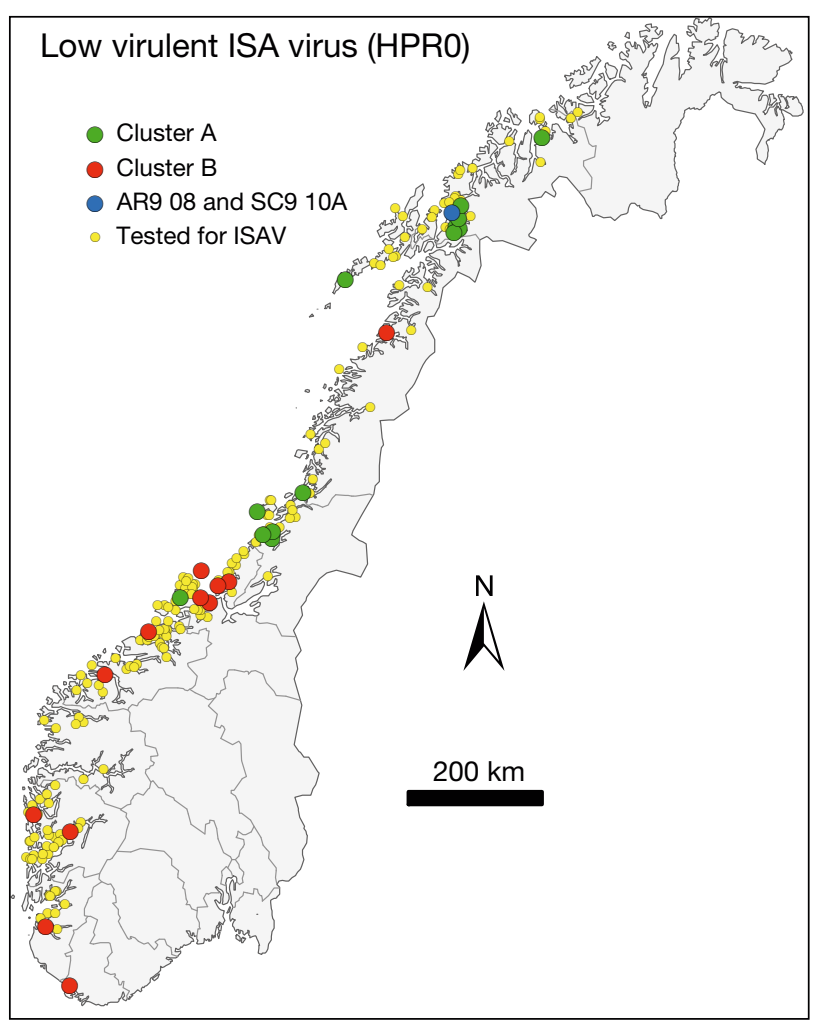

Fig. 1. Farm sites (yellow) in Norway hosting groups of Atlantic salmon that were tested for infectious salmon anaemia virus (ISAV) in the study period (2006 to 2010). The geographical locations for salmon groups with low virulent ISAV (ISAV-HPR0) are shown. Clusters A (green) and B (red) correspond to Clusters A and B in Fig. 2. AR9/08 and

SC9/10A represent the sequences also shown in Fig. 2

sites in Norway. The samples consisted of gill tissue, heart tissue, kidney tissue or a mix of organs. A median number of 30 fish (5th and 95th percentiles $=16$ and 66) were sampled per fish group (Table 1).

Since we were aiming to test whether ISAV-HPR0 could be the ancestor of virulent ISAV, we were only interested in 'isolated ISA outbreaks'. In this paper, isolated ISA outbreaks were defined as outbreaks that were geographically and/or genetically isolated from any known source of infectious fish. We used a stochastic model to identify 17 isolated ISA outbreaks from January 2006 to August 2009 (Aldrin et al. 2011). For these 17 outbreaks, the estimated probability of infection originating from an adjacent ISA outbreak was less than $10 \%$ in the stochastic model, implying that the source of infection was unknown for these outbreaks. Using a similar argument, 4 ISA outbreaks from the period September 2009 to December 2010 were additionally identified as isolated ISA outbreaks. Altogether, the outbreak data included HE gene sequences from all 21 isolated ISA
Table 1. Results from screening of tissue samples for infectious salmon anaemia virus (ISAV). The samples originated from groups of Atlantic salmon Salmo salar consisting of (1) juveniles in the freshwater phase (which can be partly exposed to seawater before transferring to a marine site), (2) on-grown marine salmon and (3) broodstock salmon. The results are listed by source (Project ID), numbers of fish groups tested for ISAV (Tested), numbers of ISAV-positive fish groups (Real-time PCR positive) and numbers of ISAVpositive fish groups with confirmed low virulent ISAV sequenced ISAV-HPR0

\begin{tabular}{|c|c|c|c|c|}
\hline \multirow[t]{2}{*}{ Category } & \multirow{2}{*}{$\begin{array}{l}\text { Project } \\
\text { ID }\end{array}$} & \multirow{2}{*}{ Tested } & \multicolumn{2}{|c|}{ No. of fish groups } \\
\hline & & & $\begin{array}{l}\text { Real-time } \\
\text { PCR positive }\end{array}$ & $\begin{array}{l}\text { Sequenced } \\
\text { ISAV-HPR0 }\end{array}$ \\
\hline \multirow[t]{3}{*}{ Juvenile } & $\mathrm{B}^{\mathrm{a}}$ & 9 & 4 & 4 \\
\hline & $\mathrm{C}^{\mathrm{b}}$ & 46 & 3 & 2 \\
\hline & $E^{b}$ & 17 & 2 & 1 \\
\hline \multirow[t]{4}{*}{ Marine } & $\mathrm{A}^{\mathrm{a}}$ & 6 & 4 & 2 \\
\hline & $\mathrm{B}^{\mathrm{a}}$ & 27 & 12 & $4(+2)^{d}$ \\
\hline & $\mathrm{D}^{\mathrm{a}}$ & 56 & 18 & $9(+2)^{d}$ \\
\hline & $\mathrm{E}^{\mathrm{b}}$ & 60 & 5 & 3 \\
\hline \multirow[t]{2}{*}{ Broodstock } & $\mathrm{A}^{\mathrm{b}, \mathrm{c}}$ & 6 & 4 & 1 \\
\hline & $\mathrm{E}^{\mathrm{b}}$ & 5 & 1 & 1 \\
\hline Total & & 232 & 53 & $27(+4)^{d}$ \\
\hline
\end{tabular}

${ }^{a}$ Mainly gill tissue

bainly organ material (kidney, heart, or mix of organs) cPooled organ samples

${ }^{\mathrm{d} N u m b e r s}$ in parentheses indicate low virulent ISAV sequences for which only partial sequences were obtained and which therefore were excluded from statistical analysis

outbreaks in farmed salmon in Norway in the period 2006 to 2010.

Geographical coordinates for the fish groups were obtained from the aquaculture register of the Directorate of Fisheries (www.fiskeridir.no/akvakultur/ registre). Data on annual numbers of fish groups transferred to marine sites (fish cohort data) were obtained from reports on production statistics (Kristoffersen et al. 2009).

\section{Laboratory investigations}

In total, 9474 salmon were screened for ISAV by real-time RT-PCR. The samples from Projects A to D (Table 1) and from the isolated ISA outbreaks were analysed for ISAV at the Norwegian Veterinary Institute using real-time RT-PCR as described in a previous study (Lyngstad et al. 2011). Samples from Project E (Table 1) were analysed for ISAV at PatoGen Analyse AS in accordance with methods described by Snow et al. (2006). Samples consisting of gill tissue 
were tested when available; otherwise, heart, kidney or tissue from a mix of organs were analysed.

The ISAV HE gene was sequenced from 1 to 3 positive fish from each of the fish groups where the virus was detected by real-time RT-PCR. Sequencing of the ISAV HE gene from positive samples with cycle threshold $(\mathrm{Ct})$ values below 34 was performed as described by Lyngstad et al. (2011).

\section{Statistical analyses}

Sequences obtained from the screening data and the outbreak data (see above) were imported into MEGA 5 Software (Tamura et al. 2011), where they were processed into 2 corresponding sets of multiple sequence alignment files (Sets I and II). Set I consisted of 36 ISAV-HPR0 HE gene sequences from 27 fish groups from the screening data, with varying numbers of sequences obtained per fish group: 1 sequence was obtained from 19 fish groups; 2 sequences from 7 groups; and 3 sequences from 1 group. Within fish groups, differences between sequences in Set I were 0 ( 3 groups); 1 (2 groups); and 2 (3 groups) nucleotides, respectively. Set II consisted of the same 36 ISAV-HPR0 HE gene sequences included in Set I and in addition 36 virulent ISAV HE gene sequences from the 21 isolated ISA outbreaks (fish groups in which ISA had been confirmed). For the isolated ISA outbreaks, 1 sequence was obtained from 6 outbreaks and 2 sequences were obtained from 15 outbreaks. Within outbreaks, differences between the sequences in Set II were 0 ( 8 outbreaks); 1 (3 outbreaks); 2 ( 2 outbreaks); 3 (1 outbreak); and 8 (1 outbreak) nucleotides, respectively. The final alignment files covered nucleotides 67 to 1138 (Set I) and 67 to 1014 (Set II), respectively, with reference to GenBank accession number EU118820. The alignment files in Set II were shorter since the HPR of the ISAV HE gene was excluded due to differential deletions in this region of the virulent ISAV HE gene sequences.

Matrices of the genetic distances between all pairs of ISAV HE gene sequences were computed for each of the 2 alignment sets using the R packages phangorn (Schliep 2011) and APE (Paradis et al. 2004, Paradis 2006, Schliep 2011). Model test was performed in the $\mathrm{R}$ package APE, comparing the substitution models raw, JC69, K80, F81, K81 and F84. The Akaike information criteria (AIC) varied from a minimum of 4583.2 to a maximum of 4584.9 for the different models. The simple Kimura 2 (K80) parameter model for nucleotide substitution was used here (Kimura 1980). When computing genetic distance between a given pair of fish groups from which we had more than 2 sequences, we selected 1 sequence if multiple sequences were identical. When not identical, the genetic distance was defined as the least possible distance between all possible pairs of sequences. There were ambiguous identities of nucleotides in 1 position in 2 sequences. Here, both alternatives (cytosine or thymine, guanine or thymine) were included when the minimum genetic distance was calculated.

Pairwise matrices of Euclidean geographic distance between sampled farms, as well as time periods between sampling dates, were also calculated.

Maximum likelihood phylogenetic analyses based on ISAV-HPR0 HE gene sequences obtained in this study, together with reference sequences obtained from GenBank were performed using PhyML (v 3.00) software (http://phylemon.bioinfo.cipf.es/phylogeny. $\mathrm{html}$ ). Clustering support was assessed through 100 bootstrap replicates. Graphic presentation of the phylogenetic tree was performed in MEGA 5 Software.

To test whether the ISAV-HPR0 sequences were genetically structured in space and time, analyses of associations between the genetic, geographic and time difference matrices were performed. The data points in our matrices containing pairwise distances are not independent; hence, a partial permutationbased Mantel test was performed using the R package Ecodist (Goslee \& Urban 2007). The p-value returned from the Mantel $r$-statistics (10000 permutations) was used to assess the significance of the Mantel correlation coefficient. In order to test whether the result was consistent, we also analysed sub-sets of the data where: (1) fish groups with juvenile salmon ( $\mathrm{n}=7$ ) were excluded; and (2) fish groups located within a large cluster of ISA outbreaks that emerged in northern Norway ( $\mathrm{n}=7$; the North-cluster, Lyngstad et al. 2011) were excluded. The sub-set representing juvenile salmon was excluded since these fish groups were land-based and had no natural contact with the marine environment. The subset representing the North-cluster could potentially skew results due to the intense sampling in the area.

Given a transition from ISAV-HPR0 to virulent ISAV (Mjaaland et al. 2002), one would expect that the 5' part of the ISAV HE gene (excluding the HPR) of virulent ISAV and its ancestral ISAV-HPR0 would show a high degree of genetic similarity. Furthermore, based on the premise that sequences from local ISAV-HPR0 are more similar than geographically distant sequences, the expectation that virulent 
ISAV should be genetically related to local ISAVHPR0 arises. A rank test was formulated to test whether ISAV HE gene sequences from isolated ISA outbreaks were sampled closer in geographic distance to the genetically most similar ISAV-HPR0 sequence than expected by chance. Genetic distances were calculated between all pairs of HE gene sequences consisting of 1 virulent ISAV sequence and 1 ISAV-HPR0 sequence using alignment Set II. Then, for each of the virulent ISAV sequences, the most similar ISAV-HPR0 sequence (minimum genetic distance) was identified, and the geographic distance between the farm sites from where this pair of sequences originated was ranked among all pairs of sequences constituting the given virulent ISAV sequence $(n=27$ sequence pairs for each virulent sequence). If there were more than 1 ISAV-HPR0 sequence with the exact same minimum genetic distance, we used the median geographic distance rank in the statistical analyses (Table A1 in Appendix 1). Finally, the sum of geographical distance ranks for all virulent ISAV sequences was compared to a simulated sum of ranks drawn randomly from a uniform distribution with minimum 1 and maximum 27 (number of ISAV-HPR0 sequences). For each iterated simulation, 37 random draws were conducted (the number of sequences in Column 3 of Table A1). In the following step, this was aggregated up to 21 ranks using the median ranks in accordance with Column 3 in Table A1. The sum of the simulated ranks was obtained for 10000 iterations and compared to the observed sum of ranks from the dataset.

The risk of having an isolated ISA outbreak in Norway was calculated as the minimum and maximum annual number of isolated ISA outbreaks divided by the mean annual number of fish cohorts transferred to the marine environment during the study period.

Statistical analyses were performed in $\mathrm{R}$ version 2.13.1 (The R Foundation for Statistical Computing) and by SAS-PC system version 9.1.3 for Windows (SAS Institute). The nucleotide sequences from this study have the accession numbers in GenBank as listed in Tables A2 \& A3 in Appendix 1.

\section{RESULTS}

ISAV was detected by real-time RT-PCR in $23 \%$ $(53 / 232)$ of the fish groups that were screened for ISAV (screening data). ISAV-HPR0 was confirmed in $59 \%(31 / 53)$ of the ISAV-positive groups, and 27 of the 31 ISAV-HPR0 sequences had enough sequence information to be included in the statistical analyses
(Table 1). Sequencing of samples from the remaining ISAV-positive fish groups was not possible due to low amounts of viral RNA (Ct-values >34).

The fish groups with ISAV-HPR0 were widely distributed along the Norwegian coast (Fig. 1). ISAV-HPR0 was detected in juvenile salmon, ongrown marine salmon and in broodstock salmon (Table 1). Three of the ISAV-HPR0-positive juvenile salmon groups had never been exposed to marine environments.

The phylogeny of the ISAV-HPR0 HE gene sequences from Norway, together with reference sequences from the Faroes, Scotland and the US, are illustrated in the phylogenetic tree (Fig. 2). Four clusters supported by high bootstrap values were designated A to D. Most of the Norwegian ISAV-HPR0 gene sequences grouped in 2 of the clusters. Cluster A contained sequences originating from fish groups mainly located in the northern part of Norway, while the sequences in Cluster B originated from fish groups located mainly in the southern part of Norway (Figs. 1 \& 2). Cluster A was further divided in 2 subgroups. The only ISAV-HPR0 sequences that did not belong to Clusters A and B were AR9_08 and SC9_10A. These were sampled on the same farm site in northern Norway but from different fish groups and 2 yr apart. Clusters C and D were composed of reference sequences from the Faroes, Scotland and the US.

The genetic distance between pairs of ISAV-HPR0 HE gene sequences and the geographic distance between the sites from where the sequences were obtained were correlated (Mantel correlation coefficient $=0.49 ; \mathrm{p}<0.002$ ). This correlation remained significant both when (1) fish groups with juvenile salmon $(n=7)$ were excluded, and when (2) fish groups located within a large cluster of ISA outbreaks that emerged in northern Norway ( $n=7$; the North-cluster, Lyngstad et al. 2011) were excluded (Mantel correlation coefficients $=0.52$ and 0.41 ; $\mathrm{p}<0.002$, respectively). Adding the time period between sampling (difference in days) did not contribute significantly to the Mantel correlation coefficient $(\mathrm{r}=0.48)$.

Virulent ISAV HE gene sequences from the 21 isolated ISA outbreaks were significantly closer in geographical distance to the genetically closest ISAVHPR0 HE gene sequences than would be expected by chance (sum of rank test, $\mathrm{p}=0.003$ ), showing that there was a spatial association between virulent ISAV and ISAV-HPR0.

Three isolated ISA outbreaks were identified in Norway in 2006, 5 in 2007, 7 in 2008, 3 in 2009, and 3 




in 2010. The mean number of fish groups transferred to marine farm sites each year in the study period was 277 (minimum 262 and maximum 290), i.e. between 1 and $3 \%$ of the fish groups experienced an isolated ISA outbreak during the marine phase.

\section{DISCUSSION}

The main results from this study were that ISAVHPR0 was frequently present in farmed populations of Atlantic salmon in Norway and that it occurs both in marine and freshwater environments, supporting previous findings (Nylund et al. 2007). We found that genetic and geographic distances between pairs of ISAV-HPR0 HE genes were positively correlated, suggesting that the population of ISAV-HPR0 is geographically structured. Finally, we found a spatial association between virulent ISAV and ISAV-HPR0, supporting the hypothesis that ancestral ISAV-HPR0 may undergo a transition to virulent ISAV.

ISAV-HPR0 was detected in Atlantic salmon both in freshwater and marine environments, and in juveniles, on-grown marine salmon and broodstock salmon. In the screening data, all positive samples that were confirmed by sequencing were identified 
as ISAV-HPR0. Low virus levels in the remaining screening samples, and no ISA suspicion reported in these fish groups, suggests that these were also ISAV-HPR0.

ISAV-HPR0 was frequently detected in the fish groups. Ct-values from previous screenings indicated markedly higher amounts of virus in gill tissues than in kidney tissues or in tissues from a mix of organs (Lyngstad et al. 2011), implying that analysing different tissue material affects the diagnostic sensitivity. Nevertheless, we did find ISAV-HPR0 in a large proportion of the 232 fish groups, and these findings were widely distributed along the Norwegian coast. Hence, this material gave an informative cross-sectional status of the geographical distribution of different genotypes of ISAV-HPR0 in the population of farmed salmon in Norway.

Numerous findings of ISAV-HPR0 have also been reported from the Faroes, where ISAV-HPR0 was detected in nearly $100 \%$ of the production cycles (Christiansen et al. 2011). The Faroese results were based on 5 to 12 samplings from all sites each year and showed that after the initial detection of ISAVHPR0 the level of detectable virus peaked after 2 mo in marine farms. There was no further detection of ISAV-HPR0 after another 2 mo (Christiansen et al. 2011). In Norway, on-grown salmon are kept at marine sites for approximately 18 mo (Kristoffersen et al. 2009). On account of the frequency with which ISAVHPR0 was detected in groups of marine salmon sampled only once (Table 1), it would not be surprising if practically all groups of marine salmon in Norway are infected with ISAV-HPR0 at some point, i.e. comparable to the Faroese findings.

The statistical analysis showed that sequences of ISAV-HPR0 from fish groups located in relative proximity were more similar than those located farther apart. Also, the phylogenetic analysis grouped the ISAV-HPR0 gene sequences in 2 main clusters: one mainly located in the southern part of Norway and one mainly in the north. The geographically structured phylogenetic pattern for ISAV-HPR0 suggests that local transmission of the virus contributes to shape local virus populations. There was no indication that the geographic structure of ISAV-HPR0 sequences was influenced by the tissue material in the samples. For example, when the analysis was limited to gill-tissue samples only, the geographic structure was retained (Mantel correlation coefficients $=0.59$; $\mathrm{p}<0.002)$. The geographic structure of ISAV-HPR0 may alternatively agree with vertical pathways of transmission (Nylund et al. 2007), horizontal transmission within farmed populations of salmon (Chris- tiansen et al. 2011) and/or involve a wild reservoir. It is not possible with the present data to distinguish among these alternatives. Nevertheless, salmon farms in Norway are usually stocked with fish from several smolt-producing sites (Lyngstad et al. 2008), and the ISAV-HPR0 detected in juvenile salmon may thus cause unintended spread of the virus when fish are transferred to marine sites (Murray et al. 2002).

If spontaneous transition of ISAV-HPR0 to virulent ISAV does occur, then the fact that the population of ISAV-HPR0 was found to be geographically structured raises the expectation that virulent ISAV should be genetically related to local ISAV-HPR0. The present rank test showed that isolated virulent ISAV-positive fish groups were located significantly closer in distance to the genetically closest ISAV-HPR0 than would be expected by chance. This result supports the hypothesis that ISA may arise from spontaneous transitions of ISAV-HPR0 to virulent ISAV (Cunningham et al. 2002, Mjaaland et al. 2002, Nylund et al. 2003). Assuming that ISAV-HPR0 occurs in $100 \%$ of the fish groups, we speculate that the probability of an isolated ISA outbreak occurring reflects the probability of transition from ISAV-HPR0 to virulent ISAV in the given fish group. The risk of such an event in a given group of fish appeared to be low. However, due to the large number of groups of farmed salmon stocked to marine farms annually, this pathway to ISA outbreaks could be an annually occurring phenomenon in Norwegian salmon farming.

With regard to associations between low virulent and virulent ISAV, some studies have suggested that possible transitions may be explained by insertion events, i.e. from virulent ISAV to ISAV-HPR0 (Kibenge et al. 2009, Castro-Nallar et al. 2011), rather than by deletion events (Mjaaland et al. 2002, Nylund et al. 2003). In the present analyses, the time period between sampling did not contribute to explain the association between ISAV-HPR0 and virulent ISAV, lending no support to either direction of hypothetical transition events. Nevertheless, deletion events are argued to be more likely to occur than inserts (Mjaaland et al. 2002, Nylund et al. 2003). It is also worth noting that all detections of ISAV in juvenile fish referred to in the present study were of the low virulent HPR0 type and, furthermore, that outbreaks of ISA have rarely been reported from juvenile salmon in the freshwater phase (Rimstad et al. 2011). Both of these lines of evidence support the hypothesis that ISAV-HPR0 may be a precursor variant from which virulent ISAV may evolve.

In conclusion, ISAV-HPR0 was detected in fish groups both in freshwater and marine environments, 
and in juvenile, on-grown marine salmon and broodstock salmon. Genetic and geographic distances between pairs of ISAV-HPR0 HE gene sequences were positively correlated, suggesting that the population of ISAV-HPR0 is geographically structured. The spatial association between fish groups with virulent and low virulent ISAV supports the hypothesis that ISAV-HPR0 may evolve to virulent ISAV, indicating that there is a risk associated with ISAV-HPR0 infection. If virulent ISAV evolves from ISAV-HPR0 in a given fish group, then neighbouring fish groups are at elevated risk (Aldrin et al. 2011, Lyngstad et al. 2011). Early detection of ISA outbreaks and implementation of measures to reduce the spread of virulent ISAV thus remain important strategies for controlling ISA.

Acknowledgements. We thank the Norwegian Food Safety Authority, and in particular E. T. Karlsen, E. J. Johansen, S. Romstad and A. Østvik (Havbrukstjenesten AS) for sampling of fish. We thank K. Haugbro and E. Trettenes for technical work in the laboratory, and E. Brun for useful discussions. The cohort study of pancreas disease (Jansen et al. 2010), the case-control study of cardiomyopathy syndrome (B. Fineid pers. comm.) and the 'Stans spredning av PD til Midt-Norge (Stop the spread of PD to Mid-Norway)' project (A. Østvik pers. comm.) are acknowledged for sharing fish samples and journal data. Finally, the Research Council of Norway and the Fishery and Aquaculture Industry Research are acknowledged for funding (project nos. 185262 and 199734/S40).

\section{LITERATURE CITED}

Aldrin M, Lyngstad TM, Kristoffersen AB, Storvik B, Borgan $\varnothing$, Jansen PA (2011) Modelling the spread of infectious salmon anaemia among salmon farms based on seaway distances between farms and genetic relationships between infectious salmon anaemia virus isolates. J R Soc Interface 8:1346-1356

> Castro-Nallar E, Cortez-San MM, Mascayano C, Molina C, Crandall KA (2011) Molecular phylodynamics and protein modeling of infectious salmon anemia virus (ISAV). BMC Evol Biol 11:349

Christiansen DH, Østergaard PS, Snow M, Dale OB, Falk K (2011) A low-pathogenic variant of infectious salmon anemia virus (ISAV-HPR0) is highly prevalent and causes a non-clinical transient infection in farmed Atlantic salmon (Salmo salar L.) in the Faroe Islands. J Gen Virol 92:909-918

Cook-Versloot M, Griffiths S, Cusack R, McGeachy S, Ritchie R (2004) Identification and characterisation of infectious salmon anaemia virus (ISAV) haemagglutinin gene highly polymorphic region (HPR) type 0 in North America. Bull Eur Assoc Fish Pathol 24:203-208

Cunningham CO, Gregory A, Black J, Simpson I, Raynard RS (2002) A novel variant of the infectious salmon anaemia virus (ISAV) haemagglutinin gene suggests mechanisms for virus diversity. Bull Eur Assoc Fish Pathol 22:366-374
Devold M, Falk K, Dale OB, Krossøy B and others (2001) Strain variation, based on the hemagglutinin gene, in Norwegian ISA virus isolates collected from 1987 to 2001: indications of recombination. Dis Aquat Org 47: 119-128

Falk K, Aspehaug V, Vlasak R, Endresen C (2004) Identification and characterization of viral structural proteins of infectious salmon anemia virus. J Virol 78:3063-3071

Goslee SC, Urban DL (2007) The ecodist package for dissimilarity-based analysis of ecological data. J Stat Softw 22: $1-19$

Jansen MD, Taksdal T, Wasmuth MA, Gjerset B and others (2010) Salmonid alphavirus (SAV) and pancreas disease (PD) in Atlantic salmon, Salmo salar L., in freshwater and seawater sites in Norway from 2006 to 2008. J Fish Dis 33:391-402

Kawaoka Y, Cox NJ, Haller O, Hongo S and others (2005) Infectious salmon anaemia virus. In: Fauquet $\mathrm{CM}$, Mayo MA, Maniloff J, Desselberger U, Ball LA (eds) Virus taxonomy. 8th report of the International Committee on Taxonomy Viruses. Elsevier Academic Press, New York, NY, p 681-693

> Kibenge FSB, Godoy MG, Wang Y, Kibenge MJT and others (2009) Infectious salmon anaemia virus (ISAV) isolated from the ISA disease outbreaks in Chile diverged from ISAV isolates from Norway around 1996 and was disseminated around 2005, based on surface glycoprotein gene sequences. Virol J 6:88

Kimura M (1980) A simple method for estimating evolutionary rates of base substitutions through comparative studies of nucleotide sequences. J Mol Evol 16:111-120

Kristoffersen AB, Viljugrein H, Kongtorp RT, Brun E, Jansen PA (2009) Risk factors for pancreas disease (PD) outbreaks in farmed Atlantic salmon and rainbow trout in Norway during 2003-2007. Prev Vet Med 90:127-136

> Krossøy B, Devold M, Sanders L, Knappskog PM and others (2001) Cloning and identification of the infectious salmon anaemia virus haemagglutinin. J Gen Virol 82:1757-1765

Lyngstad TM, Jansen PA, Sindre H, Jonassen CM, Hjortaas MJ, Johnsen S, Brun E (2008) Epidemiological investigation of infectious salmon anaemia (ISA) outbreaks in Norway 2003-2005. Prev Vet Med 84:213-227

> Lyngstad TM, Hjortaas MJ, Kristoffersen AB, Markussen T, Karlsen ET, Jonassen CM, Jansen PA (2011) Use of molecular epidemiology to trace transmission pathways for infectious salmon anaemia virus (ISAV) in Norwegian salmon farming. Epidemics 3:1-11

$>$ McBeath AJA, Bain N, Snow M (2009) Surveillance for infectious salmon anaemia virus HPR0 in marine Atlantic salmon farms across Scotland. Dis Aquat Org 87:161-169

- Mjaaland S, Hungnes O, Teig A, Dannevig BH, Thorud K, Rimstad E (2002) Polymorphism in the infectious salmon anemia virus hemagglutinin gene: importance and possible implications for evolution and ecology of infectious salmon anemia disease. Virology 304:379-391

> Mjaaland S, Rimstad E, Falk K, Dannevig BH (1997) Genomic characterization of the virus causing infectious salmon anemia in Atlantic salmon (Salmo salar L.): an orthomyxo-like virus in a teleost. J Virol 71:7681-7686

> Murray AG, Smith RJ, Stagg RM (2002) Shipping and the spread of infectious salmon anemia in Scottish aquaculture. Emerg Infect Dis 8:1-5

Norwegian Food Safety Authority (2007) Contingency plan for control of ISA in Norway. Available at www. mattilsynet.no/mattilsynet/multimedia/archive/00025/ 
ILA_contingensy_plan_25394a.pdf (accessed on 1 November 2012)

Nylund A, Devold M, Plarre H, Isdal E, Aarseth M (2003) Emergence and maintenance of infectious salmon anaemia virus (ISAV) in Europe: a new hypothesis. Dis Aquat Org 56:11-24

Nylund A, Plarre H, Karlsen M, Fridell F, Ottem KF, Bratland A, Saether PA (2007) Transmission of infectious salmon anaemia virus (ISAV) in farmed populations of Atlantic salmon (Salmo salar). Arch Virol 152:151-179

Office International des Epizooties (2009) Infectious salmon anaemia. Available at www.oie.int/en/internationalstandard-setting/aquatic-manual/access-online/ (accessed on 25 January 2012)

Paradis E (2006) Analyses of phylogenetics and evolution with R. Springer, New York, NY

Paradis E, Claude J, Strimmer K (2004) APE: analyses of phylogenetics and evolution in R language. Bioinformatics 20:289-290

Plarre H, Devold M, Snow M, Nylund A (2005) Prevalence of infectious salmon anaemia virus (ISAV) in wild salmonids in western Norway. Dis Aquat Org 66:71-79

Raynard RS, Murray AG, Gregory A (2001) Infectious salmon anaemia virus in wild fish from Scotland. Dis Aquat Org 46:93-100

Rimstad E, Mjaaland S, Snow M, Mikalsen AB, Cunningham CO (2001) Characterization of the infectious salmon anemia virus genomic segment that encodes the putative hemagglutinin. J Virol 75:5352-5356

Rimstad E, Dale OB, Dannevig BH, Falk K (2011) Infectious salmon anaemia. In: Woo PTK, Bruno DW (eds) Fish diseases and disorders, Vol 3. Viral, bacterial and fungal infections, 2nd edn. CAB International, Wallingford, p 143-165

Schliep KP (2011) phangorn: phylogenetic analysis in R. Bioinformatics 27:592-593

Snow M, McKay P, McBeath AJ, Black J and others (2006) Development, application and validation of a Taqman real-time RT-PCR assay for the detection of infectious salmon anaemia virus (ISAV) in Atlantic salmon (Salmo salar). Dev Biol 126:133-145

- Tamura K, Peterson D, Peterson N, Stecher G, Nei M, Kumar S (2011) MEGA 5: molecular evoutionary genetics analysis using maximum likelihood, evolutionary distance, and maximum parsimony methods. Mol Biol Evol 28: $2731-2739$

Appendix 1. Tables on associations between virulent and low virulent infectious salmon anaemia virus (ISAV) sequences (Table A1), and GenBank accession numbers for the ISAV sequences in the study (Tables A2 and A3)

Table A1. Each of 21 isolated infectious salmon anaemia (ISA) outbreaks tabulated with the genetic distance to the most similar ISAV-HPR0 sequence; the number of ISAV-HPR0 sequences with this exact minimum genetic distance; geographic distance between the given ISA outbreak farm site and the farm site where the most similar ISAV-HPR0 sequence originated; and the rank of geographic distance to the most similar ISAV-HPR0 sequence. When the number of ISAV-HPR0 sequences is $>1$, geographic distance and distance ranks are given as medians (minimum and maximum values)

\begin{tabular}{|lcccc|}
\hline $\begin{array}{l}\text { Isolated ISA } \\
\text { outbreak no. }\end{array}$ & $\begin{array}{c}\text { Genetic } \\
\text { distance }\end{array}$ & $\begin{array}{c}\text { No. of } \\
\text { ISAV-HPR0 } \\
\text { sequences }\end{array}$ & $\begin{array}{c}\text { Geographic } \\
\text { distance, km } \\
\text { (min.-max.) }\end{array}$ & $\begin{array}{c}\text { Rank geographic } \\
\text { distance } \\
\text { (min.-max.) }\end{array}$ \\
\hline 1 & 0.002 & 4 & $23.5(11.9-523.1)$ & $2.5(1-18)$ \\
2 & 0 & 1 & 883.6 & 16 \\
3 & 0 & 4 & $262.8(253.0-282.6)$ & $6.5(5-9)$ \\
4 & 0.001 & 1 & 893.7 & 24 \\
5 & 0.013 & 1 & 834.3 & 24.5 \\
6 & 0.001 & 1 & 241.4 & 10 \\
7 & 0.001 & 4 & $51.9(41.1-585.3)$ & $3.5(1-20)$ \\
8 & 0 & 4 & $45.5(34.2-501.7)$ & $3.5(1-18)$ \\
9 & 0.007 & 4 & $19.8(8.5-527.4)$ & $2.5(1-18)$ \\
10 & 0 & 1 & 4.7 & 1 \\
11 & 0.001 & 1 & 29.5 & 1 \\
12 & 0.001 & 1 & 140.9 & 3 \\
13 & 0.010 & 1 & 229.2 & 7 \\
14 & 0 & 1 & 25.3 & 22 \\
15 & 0 & 1 & 801.2 & 1.5 \\
16 & 0.002 & 1 & 82.7 & 16 \\
17 & 0.001 & 1 & 832.8 & 22 \\
18 & 0.007 & 1 & 963.9 & 1.5 \\
19 & 0.010 & 1 & 30.8 & 24.5 \\
20 & 0.012 & 1 & 681.5 & $8.5(8-10)$ \\
21 & 0.005 & 2 & $291.8(207.2-376.4)$ & \\
\hline
\end{tabular}


Table A2. Site identities (ID) of the nucleotide sequences from the screening dataset, GenBank accession numbers, and reference to the corresponding clusters in Figs. 1 \& 2

\begin{tabular}{|c|c|c|}
\hline ID & GenBank accession no. & Cluster \\
\hline AR2 08 & FN687348 & $\mathrm{A}$ \\
\hline AR4 08 & HE800144 & A \\
\hline AR7 08 & FN687351 & A \\
\hline AR8 08 & FN687352 & $\mathrm{A}$ \\
\hline AR26 08A & FN687356 & \\
\hline AR26 08B & FN687357 & $\mathrm{A}$ \\
\hline AR9 08 & FN687353 & \\
\hline SC1 06 & HE800145 & B \\
\hline $\mathrm{SC} 206$ & HE800146 & B \\
\hline SC1 08A & HE800147 & \\
\hline SC1 08B & HE800148 & B \\
\hline SC1 09 & HE800149 & B \\
\hline SC2 09B & HE800150 & $\mathrm{A}$ \\
\hline $\mathrm{SC} 209 \mathrm{C}$ & HE800151 & \\
\hline SC3 09A & HE800152 & B \\
\hline SC3 09B & HE800153 & \\
\hline SC1 10 & HE800154 & $\mathrm{A}$ \\
\hline SC2 10A & HE800155 & A \\
\hline SC2 10B & HE800156 & \\
\hline SC4 10 & HE800157 & B \\
\hline SC5 10 & HE800158 & B \\
\hline SC6 10 & HE800159 & B \\
\hline SC7 10 & HE800160 & B \\
\hline SC9 10A & HE800161 & \\
\hline SC10 10A & HE800162 & A \\
\hline SC11 10 & HE800163 & $\mathrm{B}$ \\
\hline SC12 10A & HE800164 & \\
\hline SC12 10B & HE800165 & B \\
\hline SC13 10 & HE800166 & A \\
\hline SC14 10A & HE800167 & B \\
\hline SC14 10B & HE800168 & \\
\hline SC15 10 & HE800169 & $\mathrm{A}$ \\
\hline SC16 10A & HE800170 & \\
\hline SC16 10B & HE800171 & A \\
\hline SC16 10C & HE800172 & \\
\hline SC17 10 & HE800173 & A \\
\hline
\end{tabular}

Editorial responsibility: Mark Crane, Geelong, Victoria, Australia
Table A3. Site identities (ID) of the nucleotide sequences from the infectious salmon anaemia (ISA) outbreak dataset and GenBank accession numbers

\begin{tabular}{|ll}
\hline ID & GenBank accession no. \\
\hline ISA1 06A & FR751546 \\
ISA1 06B & FR751547 \\
ISA2 06A & FR751548 \\
ISA2 06B & FR751549 \\
ISA3 06A & FR751550 \\
ISA3 06B & FR751551 \\
ISA1 07 & FN687284 \\
ISA2 07A & FN687286 \\
ISA2 07B & FN687287 \\
ISA3 07A & FN687288 \\
ISA3 07B & FN687289 \\
ISA4 07A & FN687290 \\
ISA4 07B & FN687291 \\
ISA7 07A & FN687303 \\
ISA7 07B & FN687304 \\
ISA1 08 & FN687305 \\
ISA3 08A & FN687308 \\
ISA3 08B & FN687309 \\
ISA4 08A & FN687310 \\
ISA4 08B & FN687311 \\
ISA5 08A & FN687312 \\
ISA5 08B & FN687313 \\
ISA8 08 & FN687318 \\
ISA9 08A & HE800180 \\
ISA9 08B & FN687319 \\
ISA4 09A & FN687320 \\
ISA4 09B & FN687333 \\
ISA8 09A & FN687340 \\
ISA9 09A & FR796471 \\
ISA4 10A & HE800174 \\
ISA4 10B & ISA5 10A \\
ISA5 10B & HE800175 \\
\hline
\end{tabular}

Submitted: March 13, 2012; Accepted: August 22, 2012

Proofs received from author(s): November 2, 2012 